\title{
Protecting the entropic uncertainty lower bound in Markovian and non-Markovian environment via additional qubits
}

\author{
S. Haseli ${ }^{1, *}$ and F. Ahmadi ${ }^{2}$ \\ ${ }^{1}$ Faculty of Physics, Urmia University of Technology, Urmia, Iran \\ ${ }^{2}$ Department of Engineering Science and Physics, Buein Zahra Technical University
}

(Dated: November 11, 2019)

\begin{abstract}
The uncertainty principle is an important principle in quantum theory. Based on this principle, it is impossible to predict the measurement outcomes of two incompatible observables, simultaneously. Uncertainty principle basically is expressed in terms of the standard deviation of the measured observables. In quantum information theory, it is shown that the uncertainty principle can be expressed by Shannon's entropy. The entopic uncertainty lower bound can be altered by considering a particle as the quantum memory which is correlated with the measured particle. We assume that the quantum memory is an open system. We also select the quantum memory from $N$ qubit which interact with common reservoir. In this work we investigate the effects of the number of additional qubits in reservoir on entropic uncertainty lower bound. We conclude that the entropic uncertainty lower bound can be protected from decoherence by increasing the number of additional qubit in reservoir.
\end{abstract}

PACS numbers:

\section{INTRODUCTION}

Uncertainty principle is one of the most fundamental and important issues in quantum theory which is determined the distinction between quantum theory and classical theory. It sets a nontrivial limit on our ability to predict the outcomes of two incompatible measurements on a quantum system. The first and well-known uncertainty relation was proposed by Heisenberg[1]. It is associated with momentum and position, measured for a particle. Based on the standard deviation of measuring outcomes of the incompatible observables position $\hat{x}$ and momentum $\hat{p}$, the Heisenberg uncertainty relation is formulated by Kennard as [2]

$$
\Delta \hat{p} \Delta \hat{x} \geq \frac{\hbar}{2}
$$

Robertson [3] and Schrodinger [4], improved the above uncertainty relation for arbitrary pairs of incompatible observables $\hat{Q}$ and $\hat{R}$ to a universal form as

$$
\Delta \hat{Q} \Delta \hat{R} \geq \frac{1}{2}|\langle\psi|[\hat{Q}, \hat{R}]| \psi\rangle|,
$$

where $\Delta \hat{O}=\sqrt{\left\langle\psi\left|\hat{O}^{2}\right| \psi\right\rangle-\langle\psi|\hat{O}| \psi\rangle^{2}}, \quad(\hat{O} \in\{\hat{Q}, \hat{R}\})$ is the standard deviations and $[\hat{Q}, \hat{R}]=\hat{Q} \hat{R}-\hat{R} \hat{Q}$. The lower bound of Robertson's uncertainty relation depends on the state of the system. It leads to a trivial result when the system is prepared in the eigenstates of anyone of the two observables. With the advent of quantum information theory, it was observed that the relation of uncertainty can be formulated in terms of Shannon entropy. The first entropic uncertainty relation EUR was suggested by Kraus [5], and then was proved by Maassen and Uffink [6]

$$
H(Q)+H(R) \geq \log _{2} \frac{1}{c},
$$

\footnotetext{
*Electronic address: soroush.haseli@uut.ac.ir
}

where $H(\hat{O})=-\sum_{o} p_{o} \log _{2} p_{o}$ is the Shannon entropy of the measured observable $\hat{O} \in\{Q, R\}, p_{o}$ is the probability of the outcome $o$, and $c=\max _{\{i, j\}}\left|\left\langle q_{i} \mid r_{j}\right\rangle\right|^{2}$ quantifies the complementarity between the observables. If the state of the measured particle is $\rho$, then the EUR can be formulated in general form as

$$
H(Q)+H(R) \geq \log _{2} \frac{1}{c}+S(\rho)
$$

where $S(\rho)=-\operatorname{tr}\left(\rho \log _{2} \rho\right)$ is the von Neumann entropy of the general state $\rho$. When the state $\rho$ is pure, the above EUR reduces to Eq. 3. In general, the EUR can be interpreted by an interesting game between two observers, Alice and Bob. At firts, Bob prepares a particle in an arbitrary quantum state $\rho$ and sends it to Alice. Then, they reach an agreement on measuring two observables $\hat{Q}$ and $\hat{R}$ by Alice on the prepared particle. Alice does her measurement on the quantum state of the prepared particle $\rho$ and declares her choice of the measurement to Bob. Bob tracks to minimize his uncertainty about the outcome of Alice measurement . The minimum of the uncertainty of Bob about Alice's measurement is bounded by Eq. 4. Let us consider the game with two particles. Bob prepares a correlated bipartite state $\rho_{A B}$ and sends one of them to Alice and the other one is kept as the quantum memory. In this game, the minimum of the uncertainty of Bob about Alice's measurement is bounded by the Quantum-memoryassisted EUR as [7]

$$
S(Q \mid B)+S(R \mid B) \geq \log _{2} \frac{1}{c}+S(A \mid B),
$$

where $S(O \mid B)=S\left(\rho^{O B}\right)-S\left(\rho^{B}\right)$ denotes the conditional von Neumann entropies of the post measurement states

$$
\rho^{O B}=\sum_{i}\left(\left|o_{i}\right\rangle\left\langle o_{i}\right| \otimes \mathbf{I}\right) \rho^{A B}\left(\left|o_{i}\right\rangle\left\langle o_{i}\right| \otimes \mathbf{I}\right),
$$

where $\left\{\mid o_{i}\right\}$ 's are the eigenstates of the observable $O$, and $\mathbf{I}$ is the identity operator. In comparison with Compared with 
Eqs. 3 and 4, one can see that the uncertainty lower bound in Eq. 5 reduces for the negative conditional entropy $S(A \mid B)$. So, Bob can guess Alice's measurement outcomes with better accuracy. Quantum-memory-assisted EUR, has a wide range of applications including entanglement detection [8-10] and quantum cryptography $[11,12]$.

Much efforts have been made to find the tighter uncertainty bounds for Quantum-memory-assisted EUR [13-22]. In Ref.[19] Adabi et al. proposed a tighter lower bound for Quantum-memory-assisted EUR. They showed that the uncertainty bound of Eq. 5 can be tightened as

$$
S(Q \mid B)+S(R \mid B) \geq \log _{2} \frac{1}{c}+S(A \mid B)+\max \{0, \delta\}
$$

where

$$
\delta=I(A ; B)-(I(Q ; B)+I(R ; B))
$$

and

$$
I(\hat{O} ; B)=S\left(\rho^{b}\right)-\sum_{i} p_{i} S\left(\rho_{i}^{B}\right)
$$

is the Holevo quantity. It shows the Bob's accessible information about Alice's measurement $\hat{O}$. When Alice measures observable $\hat{O}$, the $i$-th outcome with probability $p_{i}=$ $\operatorname{tr}_{A B}\left(\Pi_{i}^{A} \rho^{A B} \Pi_{i}^{A}\right)$ is obtained and Bob state is left in the corresponding state $\rho_{i}^{B}=\frac{\operatorname{tr} A\left(\Pi_{i}^{A} \rho^{A B} \Pi_{i}^{A}\right)}{p_{i}}$. Adabi's uncertainty bound given on the right-hand side (RHS) of Eq. 7 is tighter than other bound which have introduced by others.

In a real sense, quantum systems interact with their surrounding subjected to information loss in the form of dissipation and decoherence. In this work we consider the case in which $N$ qubits interacts with common environment. We select the quantum memory from these $N$ qubits. We investigate how these additional qubits effect on entropic uncertainty lower bound EULB. The work is organized as follow. In Sec. II, We will review the dynamical model which is used in this work. We will examine some examples in Sec. III. The manuscript will close with a conclusion in Sec. IV.

\section{DYNAMICAL MODEL}

Let us consider $N$ single-qubit which are located in a common dissipative reservoir. We suppose that each qubit is independently coupled to common zero temperature thermal reservoir that is consist of harmonic oscillators. The Hamiltonian of the total system ( $N$ single qubit + reservoir) is given by $[23,24]$

$$
\begin{aligned}
H & =\hat{H}_{0}+\hat{H}_{I} \\
& =\omega_{0} \sum_{i=1}^{N} \sigma_{i}^{+} \sigma_{i}^{-}+\sum_{k} \omega_{k} b_{k}^{\dagger} b_{k} \\
& +\sum_{i=1}^{k} \sum_{k}\left(g_{k}^{\star} b_{k}^{\dagger} \sigma_{i}^{-}+g_{k} b_{k} \sigma_{i}^{+}\right),
\end{aligned}
$$

where $g_{k}$ 's are the coupling strength between the $i^{\text {th }}$ qubit with transition frequency $\omega_{0}$ and $k^{t h}$ field mode with frequency $\omega_{k}, b_{k}^{\dagger}$ and $b_{k}$ are creation and annihilation operators of the $k^{t h}$ field mode, respectively. $\sigma_{i}^{+}$and $\sigma_{i}^{-}$are the $i^{t h}$ qubit raising and lowering operators, respectively. We consider the case in which there exist one excitation in the total system and reservoir in the vacuum state $|0\rangle_{E}$, initially. We also suppose that the initial state of the whole system is given by

$$
|\psi(0)\rangle=C_{0}(0)|0\rangle_{s} \otimes|0\rangle_{E}+\sum_{i=1}^{N} C_{i}(0)|i\rangle_{s} \otimes|0\rangle_{E},
$$

where $|0\rangle_{s}$ means that all qubits are in ground state $|0\rangle$, and $|i\rangle_{s}$ shows that $i^{t h}$ qubit in the excited state $|1\rangle$ and the others are in ground state $|0\rangle$. The dynamics of the whole system can be written as

$$
\begin{aligned}
|\psi(t)\rangle & =\left(C_{0}(t)|0\rangle_{s}+\sum_{i=1}^{N} C_{i}(t)|i\rangle_{s}\right) \otimes|0\rangle_{E} \\
& +\sum_{j=1}^{N} C_{j}(t)|0\rangle_{s} \otimes\left|1_{j}\right\rangle_{E},
\end{aligned}
$$

where $\left|1_{j}\right\rangle_{E}$ is the state of the reservoir with single excitation in the $j^{\text {th }}$ field mode. The Schrodinger equation in the interaction picture has the form

$$
i \frac{d}{d t}|\psi(t)\rangle=\hat{H}_{I}(t)|\psi(t)\rangle
$$

where $\hat{H}_{I}(t)=e^{i \hat{H}_{0} t} \hat{H}_{I} e^{-i \hat{H}_{0} t}$. By solving Eq. (13) and following the method which has outlined in Ref. [24], the dynamis of the $i^{\text {th }}$ qubit can be obtained as

$$
\rho_{i}(t)=\left(\begin{array}{cc}
\rho_{00}^{i}\left|C_{i}(t)\right|^{2} & \rho_{01}^{i} C_{i}(t) \\
\rho_{10}^{i} C_{i}^{\star}(t) & 1-\rho_{00}^{i}\left|C_{i}(t)\right|^{2}
\end{array}\right) .
$$

From Eq. (13), we have $\dot{C}_{0}(t)=0$ and so $C_{0}(t)=C_{0}(0)=$ $C_{0}$. The other probability coefficients satisfies the following integro-differential equations

$$
\frac{d}{d t} C_{i}(t)=-\int_{0}^{\infty} \int_{-\infty}^{+\infty} J(\omega) e^{i\left(\omega_{0}-\omega_{k}\right)(t-\tau)} \sum_{j=1}^{N} C_{j}(\tau) d \omega d \tau
$$

where $J(\omega)$ is the spectral density of the reservoir. Let us consider a Lorantzian spectral density

$$
J(\omega)=\frac{1}{2 \pi} \frac{\gamma_{0} \lambda^{2}}{\left(\omega_{0}-\omega\right)^{2}+\lambda^{2}}
$$

where the spectral width of the coupling $\lambda$ is related to the correlation time of the environment $\tau_{E}$ via $\tau_{E} \simeq 1 / \lambda$. The parameter $\gamma_{0}$ is connected to the relaxation time $\tau_{E}$, over which the state of the system changes, by $\tau_{E} \simeq 1 / \gamma_{0}$. If $\gamma_{0} / \lambda \leq 1 / 2$ we have the weak system-reservoir coupling regime and the dynamic is Markovian, while for $\gamma_{0} / \lambda>1 / 2$, we have the 


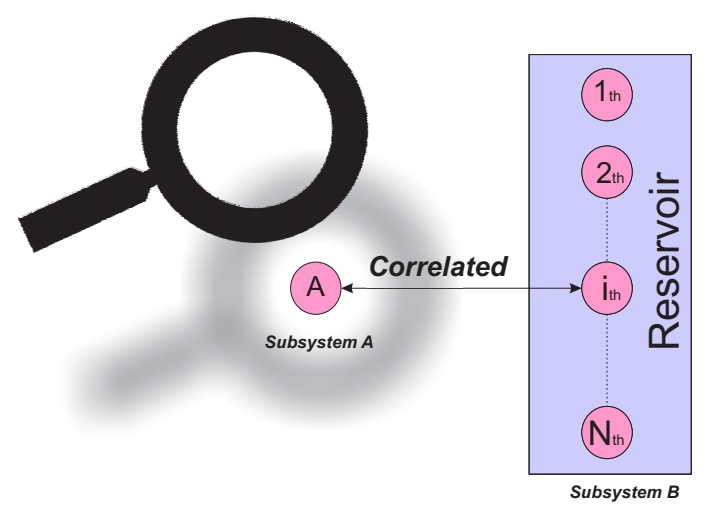

Figure 1: Schematic representation of the model where quantum memory interacts with environment. While Alice performs measurement on her particle.

strong coupling regime and the dynamic is on-Markovian. Using Laplace transformation and its inverse, the probability coefficient $C_{i}(t)$ can be obtained as

$$
C_{i}(t)=\frac{N-1}{N}+\frac{e^{-\lambda t / 2}}{N}\left[\cosh \left(\frac{D t}{2}\right)+\frac{\lambda}{D} \sinh \left(\frac{D t}{2}\right)\right],
$$

where $D=\sqrt{\lambda^{2}-2 N \gamma_{0} \lambda}$.

\section{EULB AND ADDITIONAL QUBITS}

In this section we use the above mentioned dynamical model to reduce the EULB in the presence of decoherence. Let us suppose that Bob prepare the initial correlated state $\rho^{A B_{i}}$ such that he chooses $i^{t h}$ single-qubit from $N$ qubits in common reservoir as the part $B$. Then, he sends particle $A$ to Alice and keeps $B$ as the quantum memory. Then, they reach an agreement on measuring observables by Alice on her particle. Alice does her measurement on the quantum state of the prepared particle and declares her choice of the measurement to Bob. Bob tracks to minimize his uncertainty about the outcome of Alice measurement .

In this model the quantum memory $B_{i}$ interacts with reservoir as an open quantum system (The model is sketched in Fig. (1)). It is expected that, as a result of the interaction between quantum memory and reservoir, the correlation between quantum memory $B$ and measured particle $A$ decreases. As mentioned before, the presence of correlation between Alice and Bob reduces the uncertainty of Bob about the outcomes of Alice's measurement. So, it is logical to expect that the EULB increases due to interaction between quantum memory and reservoir.

\section{A. Examples}

Maximally entangled state: Let us consider the case in which Bob prepares a maximally entangled pure state
$|\phi\rangle_{A B_{i}}=1 / \sqrt{2}\left(|00\rangle_{A B_{i}}+|11\rangle_{A B_{i}}\right)$. If the quantum memory $B_{i}$ interacts with environment, the dynamic of the bipartite quantum state $\rho_{A B_{i}}=|\phi\rangle_{A B_{i}}\langle\phi|$ can be obtained as

$$
\rho_{i}(t)=\frac{1}{2}\left(\begin{array}{cccc}
\left|C_{i}(t)\right|^{2} & 0 & 0 & C_{i}(t) \\
0 & 1-\left|C_{i}(t)\right|^{2} & 0 & 0 \\
0 & 0 & 0 & 0 \\
C_{i}^{\star}(t) & 0 & 0 & 1
\end{array}\right) .
$$

Alice and Bob reach an agreement on measuring two observables $\hat{\sigma_{x}}$ and $\hat{\sigma_{z}}$. The von Neumann entropy of the post measurement states are given by

$$
\begin{aligned}
S\left(\rho_{\hat{\sigma_{x}} B_{i}}\right) & =-\frac{1-\eta}{2} \log _{2}\left(\frac{1-\eta}{4}\right)-\frac{1+\eta}{2} \log _{2}\left(\frac{1+\eta}{4}(1) 9\right) \\
S\left(\rho_{\hat{\sigma_{z}} B_{i}}\right) & =\frac{1}{2}-\frac{\left|C_{i}(t)\right|^{2}}{2} \log _{2} \frac{\left|C_{i}(t)\right|^{2}}{2} \\
& -\frac{1-\left|C_{i}(t)\right|^{2}}{2} \log _{2} \frac{1-\left|C_{i}(t)\right|^{2}}{2},
\end{aligned}
$$

where $\eta=\sqrt{1-\left|C_{i}(t)\right|^{2}+\left|C_{i}(t)\right|^{4}}$. So, the left-hand side (LHS) of Eq.(7) is obtained as

$$
\begin{aligned}
U_{L} & =\frac{1}{2}-\frac{1-\eta}{2} \log _{2}\left(\frac{1-\eta}{4}\right)-\frac{1+\eta}{2} \log _{2}\left(\frac{1+\eta}{4}\right) \\
& -\frac{\left|C_{i}(t)\right|^{2}}{2} \log _{2} \frac{\left|C_{i}(t)\right|^{2}}{2}-\frac{1-\left|C_{i}(t)\right|^{2}}{2} \log _{2} \frac{1-\left|C_{i}(t)\right|^{2}}{2} \\
& -S_{\text {bin }}\left(\frac{\left|C_{i}(t)\right|^{2}}{2}\right)
\end{aligned}
$$

where $S_{b i n}(x)=-x \log _{2} x-(1-x) \log _{2}(1-x)$. The righthand side (RHS) of Eq.(7) is given by

$$
U_{R}=1+S_{b i n}\left(\frac{1-\left|C_{i}(t)\right|^{2}}{2}\right)-S_{b i n}\left(\frac{\left|C_{i}(t)\right|^{2}}{2}\right)+\max \{0, \delta\},
$$

where

$$
\begin{aligned}
\delta & =-\frac{1}{2}-\frac{1-\eta}{2} \log _{2}\left(\frac{1-\eta}{4}\right)-\frac{1+\eta}{2} \log _{2}\left(\frac{1+\eta}{4}\right) \\
& -\frac{\left|C_{i}(t)\right|^{2}}{2} \log _{2} \frac{\left|C_{i}(t)\right|^{2}}{2}-\frac{1-\left|C_{i}(t)\right|^{2}}{2} \log _{2} \frac{1-\left|C_{i}(t)\right|^{2}}{2} \\
& -S_{b i n}\left(\frac{1-\left|C_{i}(t)\right|^{2}}{2}\right)-S_{b i n}\left(\frac{\left|C_{i}(t)\right|^{2}}{2}\right)
\end{aligned}
$$

In Fig. 2, the EULB, $U_{R}$ is plotted as a function of time in non-Markovian regime $\lambda=0.1 \gamma_{0}$ for various number of additional qubit $N$. As can be seen from Fig. 2, due to the interaction between quantum memory and reservoir, EULB is increased through time. One can see, the lower bound decreases by increasing the number of additional qubit. In Fig. $3, U_{R}$ is plotted as a function of time in Markovian regime $\lambda=40 \gamma_{0}$ for various number of additional qubits $N$. As can be seen from Fig. 4, $U_{R}$ is increased over time, while it is decreased by increasing the number of additional qubits.

Bell diagonal state: As the second example, let us consider the set of two-qubit states with the maximally mixed marginal states. This state can be written as

$$
\rho_{A B_{i}}=\frac{1}{4}\left(\mathbf{I} \otimes \mathbf{I}+\sum_{i=1}^{3} r_{i} \sigma_{i} \otimes \sigma_{i}\right)
$$




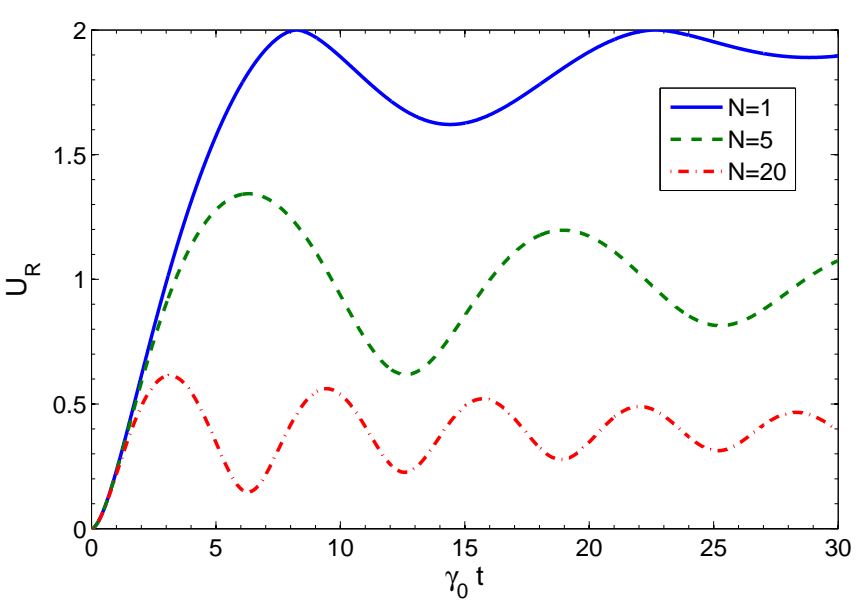

Figure 2: Lower bounds of the entropic uncertainty relation of the two complementary observables $\hat{\sigma}_{x}$ and $\hat{\sigma}_{z}$ as a function of $\gamma_{0} t$, when Bob prepare a maximally entangled state $|\phi\rangle=1 / \sqrt{2}(|00\rangle+$ $|11\rangle)$ in non-Markovian regime $\lambda=0.1 \gamma_{0}$.

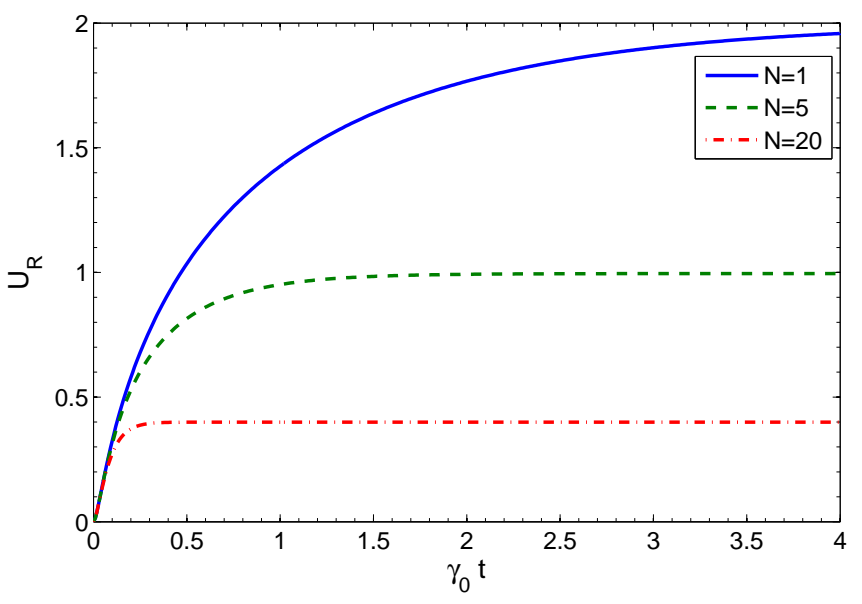

Figure 3: Lower bounds of the entropic uncertainty relation of the two complementary observables $\hat{\sigma}_{x}$ and $\hat{\sigma}_{z}$ as a function of $\gamma_{0} t$, when Bob prepare a maximally entangled state $|\phi\rangle=1 / \sqrt{2}(|00\rangle+$ $|11\rangle)$ in Markovian regime $\lambda=40 \gamma_{0}$.

where $\sigma_{i}$ 's are Pauli matrices and $\vec{r}=\left(r_{1}, r_{2}, r_{3}\right)$ belongs to a tetrahedron defined by the set of $(-1,-1,-1),(-1,1,1)$, $(1,-1,1)$ and $(1,1,-1)$. Bob prepares the state with $r_{1}=$ $1-2 p, r_{2}=r_{3}=-p$, such that the state in Eq. 24 becomes

$$
\rho^{A B_{i}}=p\left|\psi^{-}\right\rangle\left\langle\psi^{-}\right|+\frac{1-p}{2}\left(\left|\psi^{+}\right\rangle\left\langle\psi^{+}|+| \phi^{+}\right\rangle\left\langle\phi^{+}\right|\right)
$$

where $\left|\phi^{ \pm}\right\rangle=\frac{1}{\sqrt{2}}[|00\rangle \pm|11\rangle]$ and $\left|\psi^{ \pm}\right\rangle=\frac{1}{\sqrt{2}}[|01\rangle \pm|10\rangle]$ are the Bell diagonal states. In the following the Bell-diagonal state with $p=1 / 2$ is considered. The dynamics of density matrix when quantum memory $B_{i}$ interacts with reservoir is

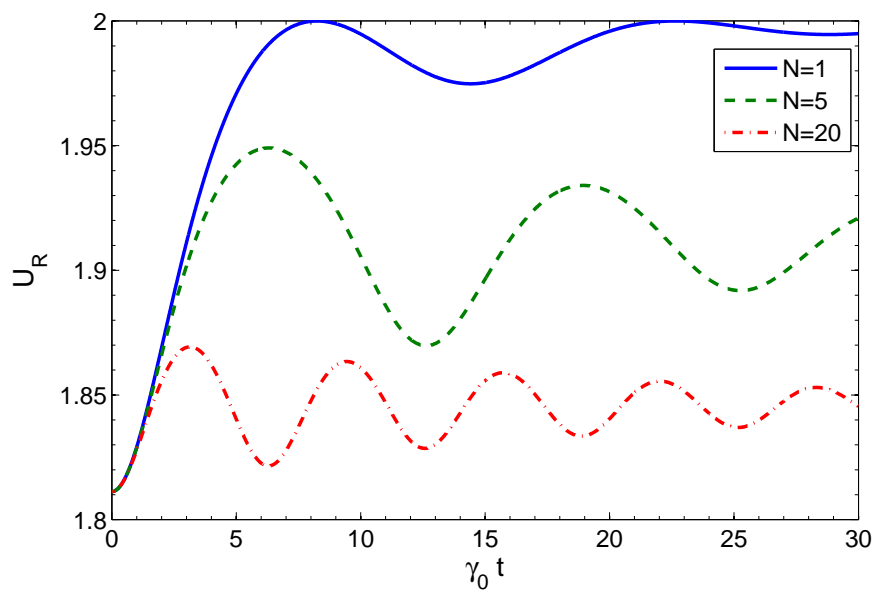

Figure 4: Lower bounds of the entropic uncertainty relation of the two complementary observables $\hat{\sigma}_{x}$ and $\hat{\sigma}_{z}$ as a function of $\gamma_{0} t$, when Bob prepare a Bell diagonal state $\rho^{A B_{i}}=p\left|\psi^{-}\right\rangle\left\langle\psi^{-}\right|+$ $\frac{1-p}{2}\left(\left|\psi^{+}\right\rangle\left\langle\psi^{+}|+| \phi^{+}\right\rangle\left\langle\phi^{+}\right|\right)$with $p=1 / 2$ in non-Markovian regime $\lambda=0.1 \gamma_{0}$.

given by

$$
\rho_{A B_{i}}=\left(\begin{array}{cccc}
\rho_{11}^{t} & 0 & 0 & \rho_{14}^{t} \\
0 & \rho_{22}^{t} & \rho_{23}^{t} & 0 \\
0 & \rho_{32}^{t} & \rho_{33}^{t} & 0 \\
\rho_{41}^{t} & 0 & 0 & \rho_{44}^{t}
\end{array}\right)
$$

where

$\rho_{11}^{t}=\frac{1+p}{4}\left|C_{i}(t)\right|^{2}, \quad \rho_{14}^{t}=\frac{1-p}{2}\left|C_{i}(t)\right|$

$\rho_{22}^{t}=\frac{1-p}{4}+\frac{1+p}{4}\left(1-\left|C_{i}(t)\right|^{2}\right), \quad \rho_{23}^{t}=\frac{1-3 p}{2} C_{i}(t)$

$\rho_{33}^{t}=\frac{1}{4}(1-p)\left|C_{i}(t)\right|^{2}, \quad \rho_{44}^{t}=\frac{1+p}{4}+\frac{1}{4}(1-p)\left(1-\left|C_{i}(t)\right|^{2}\right)$.

Alice and Bob reach an agreement on measuring two observables $\hat{\sigma_{x}}$ and $\hat{\sigma_{z}}$. The von Neumann entropy of the post measurement states are given by

$$
\begin{aligned}
S\left(\rho_{\hat{\sigma_{x}} B_{i}}\right) & =-\frac{\left|C_{i}(t)\right|^{2}}{2} \log _{2} \frac{\left|C_{i}(t)\right|^{2}}{4} \\
& -\frac{2-\left|C_{i}(t)\right|^{2}}{2} \log _{2} \frac{2-\left|C_{i}(t)\right|^{2}}{4} \\
S\left(\rho_{\hat{\sigma_{z} B_{i}}}\right) & =-\frac{\left|C_{i}(t)\right|^{2}}{8} \log _{2} \frac{\left|C_{i}(t)\right|^{2}}{8}-\frac{3\left|C_{i}(t)\right|^{2}}{8} \log _{2} \frac{3\left|C_{i}(t)\right|^{2}}{8} \\
& -\frac{4-3\left|C_{i}(t)\right|^{2}}{8} \log _{2} \frac{4-3\left|C_{i}(t)\right|^{2}}{8} \\
& -\frac{4-\left|C_{i}(t)\right|^{2}}{8} \log _{2} \frac{4-\left|C_{i}(t)\right|^{2}}{8}
\end{aligned}
$$




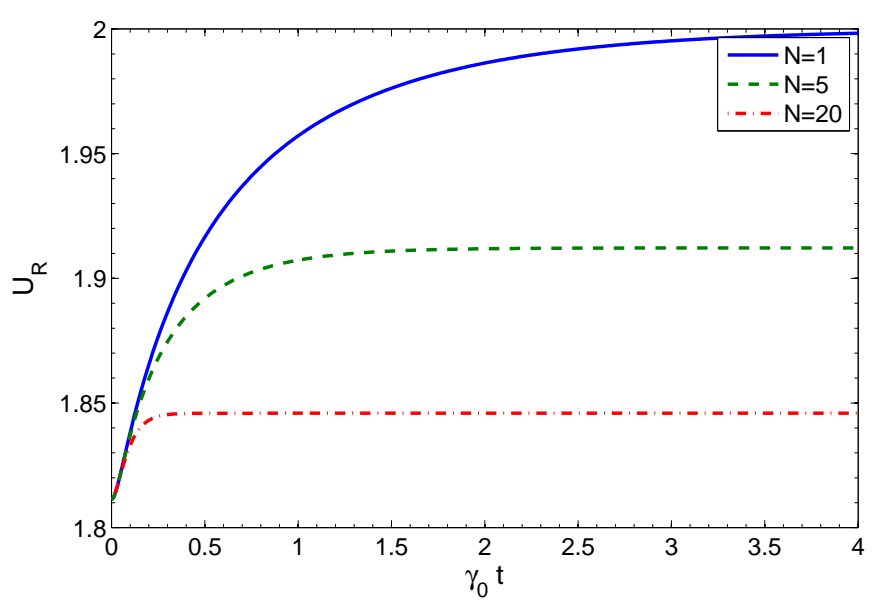

Figure 5: Lower bounds of the entropic uncertainty relation of the two complementary observables $\hat{\sigma}_{x}$ and $\hat{\sigma}_{z}$ as a function of $\gamma_{0} t$, when Bob prepare a Bell diagonal state $\mathrm{s} \rho^{A B_{i}}=p\left|\psi^{-}\right\rangle\left\langle\psi^{-}\right|+$ $\frac{1-p}{2}\left(\left|\psi^{+}\right\rangle\left\langle\psi^{+}|+| \phi^{+}\right\rangle\left\langle\phi^{+}\right|\right)$with $p=1 / 2$ in Markovian regime $\lambda=40 \gamma_{0}$.

So, the left-hand side (LHS) of Eq.(7) is obtained as where

$$
\begin{aligned}
\alpha_{ \pm} & =\left(2 \pm\left|C_{i}(t)\right|^{2}\right) / 2 \\
\theta & =\left(\sqrt{1-\left|C_{I} t\right|^{2}+\left|C_{i}(t)\right|^{4}}\right) / 4
\end{aligned}
$$

and

$$
\begin{aligned}
\delta & =\left(\alpha_{-}-\theta\right) \log _{2}\left(\alpha_{-}-\theta\right)+\left(\alpha_{+}-\theta\right) \log _{2}\left(\alpha_{+}-\theta\right) \\
& +\left(\alpha_{-}+\theta\right) \log _{2}\left(\alpha_{-}+\theta\right)+\left(\alpha_{+}+\theta\right) \log _{2}\left(\alpha_{+}+\theta\right) \\
& -S_{b i n}\left(\frac{\left|C_{i}(t)\right|^{2}}{2}\right)+S\left(\rho_{\hat{\sigma_{z}} B_{i}}\right)+S\left(\rho_{\hat{\sigma_{x}} B_{i}}\right)
\end{aligned}
$$

In Fig. 4, the EULB, $U_{R}$ is plotted as a function of time in non-Markovian regime $\lambda=0.1 \gamma_{0}$ for various number of additional qubit $N$. As can be seen from Fig. 4, due to the interaction between quantum memory and reservoir, EULB is increased through time. One can see, the lower bound decreases by increasing the number of additional qubit. In Fig. $5, U_{R}$ is plotted as a function of time in Markovian regime $\lambda=40 \gamma_{0}$ for various number of additional qubits $N$. As can be seen from Fig. 5, $U_{R}$ is increased over time, while it is decreased by increasing the number of additional qubits.

$$
\begin{aligned}
U_{L} & =-\frac{\left|C_{i}(t)\right|^{2}}{2} \log _{2} \frac{\left|C_{i}(t)\right|^{2}}{4} \\
& -\frac{2-\left|C_{i}(t)\right|^{2}}{2} \log _{2} \frac{2-\left|C_{i}(t)\right|^{2}}{4}-\frac{\left|C_{i}(t)\right|^{2}}{8} \log _{2} \frac{\left|C_{i}(t)\right|^{2}}{8}
\end{aligned}
$$
model we have considered here is such that the quantum mem-

(28dry along with $N-1$ non-interacting qubits located in com-

\section{CONCLUSION} mon reservoir. We assume that these $N$ qubit independently are coupled to a common reservoir. It is logical to expect that the EULB increases over time. However, we have shown that the EULB can be protected from decoherence by controlling the additional qubits in reservoir. It has been shown that for both Markovian and non-Markovian regime the EULB is decreased by increasing the number of additional qubits.
[1] W. Heisenberg, Z. Phys.,43 173 (1927).

[2] E. H. Kennard, Z. Phys.,44 326 (1927).

[3] H.P. Robertson : The uncertainty principle. Phys. Rev. 34, 163 (1929).

[4] E. Schrödinger, : About Heisenberg uncertainty relation. Proc. Pruss. Acad. Sci. XIX, 296 (1930).

[5] K. Kraus, Phys. Rev. D 35, 3070 (1987).

[6] H. Maassen and J. B. M. Uffink, Phys. Rev. Lett. 60, 1103 (1988).

[7] M. Berta,M. Christandl, R. Colbeck, J.M. Renes, andR.Renner, Nat. Phys. 6, 659 (2010).

[8] Y. Huang, Phys. Rev. A 82, 012335 (2010).

[9] R. Prevedel, D. R. Hamel, R. Colbeck, K. Fisher and K. J. Resch, Nature Phys. 7, 757 (2011).

[10] L. Chuan-Feng, J.-S.Xu, X.-Y.Xu, K. Li and G.-C.Guo, Nature
Phys. 7, 752 (2011).

[11] M. Tomamichel, C. C. W. Lim, N. Gisin and R. Renner, Nature Commun. 3, 634 (2012).

[12] N. H. Y. Ng, M. Berta and S. Wehner, Phys. Rev. A 86, 042315 (2012).

[13] A. K. Pati, M. M.Wilde, A. R. Usha Devi, A. K. Rajagopal and Sudha, Phys. Rev. A 86, 042105 (2012).

[14] T. Pramanik, P. Chowdhury, and A. S. Majumdar, Phys. Rev. Lett. 110, 020402 (2013).

[15] P. J. Coles and M. Piani, Phys. Rev. A 89, 022112 (2014).

[16] S. Liu, L.-Z. Mu, H. Fan, Phys. Rev. A 91, 042133 (2015).

[17] J. Zhang, Y. Zhang and C-s. Yu, Sci Rep. 5, 11701 (2015).

[18] T. Pramanik, S. Mal and A. S. Majumdar, Quantum Inf. Process. 15, 981 (2016).

[19] F. Adabi, S. Salimi, S. Haseli, Phy. Rev. A 93 (6), 062123 
(2016).

[20] F. Adabi, S. Salimi, S. Haseli,EPL (Europhysics Letters) 115 (6), 60004 (2016).

[21] H Dolatkhah, S Haseli, S Salimi, SA Khorashad, Quant. Inf. Process. 18:13 (2019).

[22] Jin-Long Huang,Wen-Cong Gan, Yunlong Xiao, Fu-Wen Shu,
Man-Hong Yung, Eur. Phys. J. C 78, 545(2018).

[23] N. Behzadi, B. Ahansaz, E. Faizi, Eur. phys. journal D 3, 80294 (2017).

[24] N. Behzadi, B. Ahansaz, A. Ektesabi, E. Faizi, Phys. Rev. A 95, 052121 (2017). 